\title{
Which lessons shall we learn from the 2019 novel coronavirus outbreak?
}

\author{
Camilla Mattiuzzi ${ }^{1}$, Giuseppe Lippi ${ }^{2}$ \\ ${ }^{1}$ Service of Clinical Governance, Provincial Agency for Social and Sanitary Services, Trento, Italy; ${ }^{2}$ Section of Clinical Biochemistry, University of \\ Verona, Verona, Italy \\ Correspondence to: Prof. Giuseppe Lippi. Section of Clinical Biochemistry, University Hospital of Verona, Piazzale LA Scuro, 37134 Verona, Italy. \\ Email: giuseppe.lippi@univr.it.
}

Submitted Feb 02, 2020. Accepted for publication Feb 04, 2020.

doi: $10.21037 / \mathrm{atm} .2020 .02 .06$

View this article at: http://dx.doi.org/10.21037/atm.2020.02.06

Two decades after the severe acute respiratory syndrome (SARS), and one decade after the middle east respiratory syndrome (MERS), a new outbreak of respiratory illness sustained by a member of the coronavirus (CoV) family has been first identified in Wuhan (Hubei Province, China), and is rapidly spreading around the world $(1,2)$. The pathogen, temporarily defined 2019 novel coronavirus (2019-nCoV), is a positive-sense RNA, 29903-bp betacoronavirus, first isolated in the Wuhan seafood market on January 7, 2020 (3), and which is highly homologous to the previous SARS CoV (4). Unlike the other two previous $\mathrm{CoV}$ zoonotic diseases caused by SARS$\mathrm{CoV}$ and MERS-CoV, transferred from bats to humans through civets and dromedary camels, respectively, it has been recently suggested that snakes may have been the intermediate reservoirs of $2019-\mathrm{nCoV}$ between bats and humans (5). The possibility of human-to-human transmission of 2019-nCoV has been documented (6), whilst it is still unclear whether the pathogen can be transmitted during the incubation period, which is apparently comprised between 2-14 days (7).

According to the last report of the World Health Organization (WHO), published on February 2, 2020, the outbreak is rapidly spreading, within and outside China (Figure 1) (8). Overall, 14,557 cases of 2019-nCoV infection have been diagnosed in 23 worldwide countries $(14,411$ cases in China, but also 20 in Japan, 19 in Thailand, 12 in Australia, 8 in the US, 8 in Germany, 6 in France and 2 in Italy, among others), 2110 of which $(15 \%)$ have been classified as severe in China. A total number of 305 deaths have been recorded so far (304 in China and 1 abroad), thus accounting for a $\sim 2.1 \%$ mortality rate (8). Despite the death rate may still be underestimated due to uncertainty on the true number of infections and the ongoing clinical progression of diseased cases, this value appears substantially lower than those earlier reported for both SARS ( 9.6\%) and MERS ( 34.4\%) (9). This evidence is in keeping with a preliminary hypothesis that the severity of this new syndrome may be lower than that of SARS and MERS, thus amplifying the likelihood of human-to-human transmission (e.g., infected people are more likely to bear mild symptoms, and hence to circulate and spread the virus) (7).

Although more data would need to be collected for accurately portend the recent $2019-\mathrm{nCoV}$ outbreak, the clinical features have been recently described in 41 Chinese patients hospitalized in Wuhan, where the infection has begun (10). Briefly, the mean age of patients was 49 (interquartile range, 41-58) years, male sex was prevalent $(73 \%)$, and nearly half of patients had an underlying pathology (20\% diabetes, $15 \%$ hypertension or cardiovascular disease). The most frequent symptoms included fever (98\%), cough $(76 \%)$, dyspnoea $(55 \%)$, myalgia (44\%), whilst headache $(8 \%)$ and diarrhoea $(3 \%)$ were less common. Notably, all patients developed pneumonia, confirmed by the presence of abnormal chest CT findings. The most frequent complications were acute respiratory distress syndrome (29\%), and acute cardiac injury (12\%). Thirty two percent of these patients ought to be admitted to the intensive care unit, whilst $15 \%$ of them died. According to the last WHO report, the clinical picture of patients diagnosed outside China is substantially similar, with a mean age of infection onset of 45 (range, 2 to 74) years, and a higher prevalence of men vs. women $(71 \%$ vs. $29 \%$ ) (8). The median period between first onset of the 


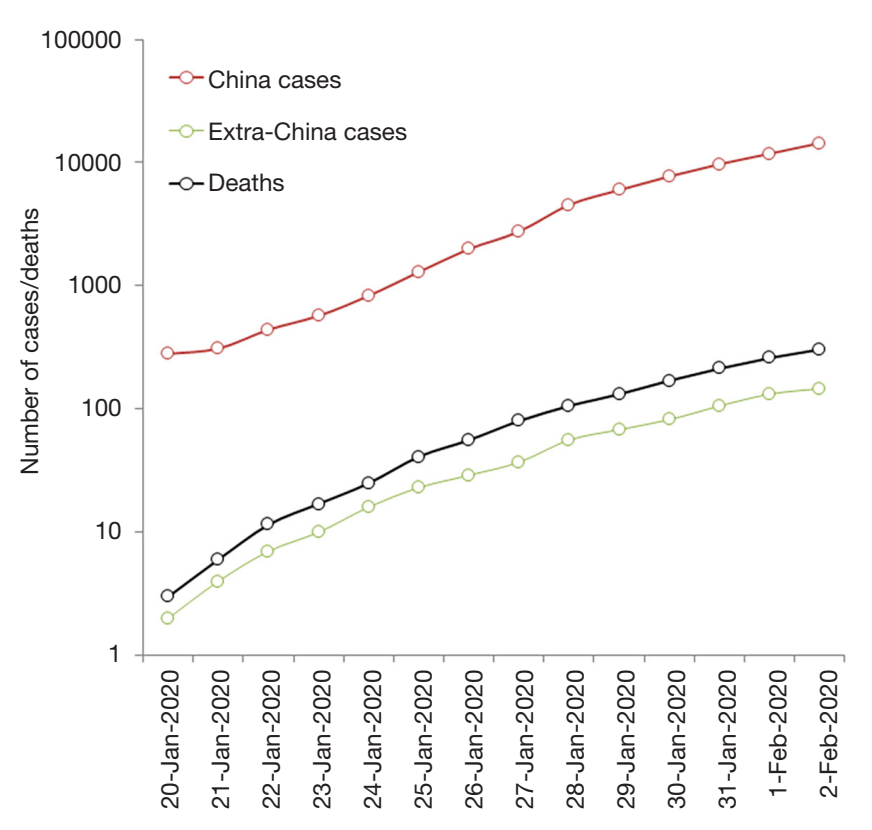

Figure 1 Current epidemics of 2019 novel coronavirus (2019$\mathrm{nCoV}$ ) infection (Source data: World Health Organization) (8).

symptoms and death has been estimated at 14 (range, 641) days, nearly half shorter in elderly people (i.e., in those aged 70-year old or older; 11 vs. 20 days; $\mathrm{P}=0.033$ ) (11). The median age of those who died for this infection appeared substantially high ( 75 years; range, $48-89$ years), and most patients (up to two-third) had important comorbidities.

An additional retrospective, single-centre study has then been published, including all confirmed 2019-nCoV cases in Wuhan Jinyintan Hospital between Jan 1 and 20, 2020 (12). The mean age of the patients was $55.5 \pm 13.1$ years, 67 were men and 32 women. Nearly half of these patients had chronic comorbidities (40\% cardiovascular diseases, $13 \%$ endocrine pathologies and $11 \%$ gastrointestinal disorders). The most frequent clinical signs and symptoms were fever (83\%), cough (82\%), dyspnea (31\%), myalgia (11\%), confusion $(9 \%)$, headache $(8 \%)$, sore throat $(5 \%)$. Less frequent symptoms were rhinorrhoea (4\%), chest pain (2\%) and diarrhoea (2\%). Overall, bilateral pneumonia was diagnosed in $75 \%$ patients, $17 \%$ developed acute respiratory distress syndrome and $11 \%$ died for multiple organ failure. Concerning laboratory data, the most frequent abnormalities were low serum albumin (98\%), increased values of C-reactive protein (86\%), erythrocyte sedimentation rate $(85 \%)$ lactate dehydrogenase $(76 \%)$ and ferritin (63\%), hyperglycaemia (52\%), increased values of interleukin 6 (IL-6; 52\%), anemia (51\%), neutrophilia (38\%), increased D-dimer values (36\%), lymphocytosis (35\%) and increased aminotransferases values (35\%). Notably, procalcitonin values were only found to be increased in $6 \%$ of all patients.

Recent information has also been published concerning the early transmission dynamics in 425 patients with confirmed infection, which has herein been defined 2019-nCoV-infected pneumonia (NCIP) (13). Briefly, more than half of the cases (i.e., $55 \%$ ) characterized by infection emergence before January 1, 2020, were linked to the Seafood Wholesale Market in Wuhan, whilst between $15-30 \%$ of cases are probably attributable to inter-human transmission. The mean incubation period was found to be 5.2 days [with a $95 \%$ confidence interval (CI) comprised between 4.1-7.0 days].

Despite the WHO has recently raised the level of global risk of this outbreak from "moderate" to "high" ("very high" in China), and many health authorities are taking prompt public health measures worldwide to face the challenge, this latest $\mathrm{CoV}$ outbreak shall now be seen as a global health concern. Urgent preventive measures shall be planned and, especially, rapid diagnostic tools shall be made available. Among the various options, it seems reasonable to conclude that amplification and detection of 2019$\mathrm{nCoV}$ using specific sequences may be preferable, since direct virus identification would then exclude the need of additional sequencing. The US Centers for Disease Control and Prevention (CDC) has recently published an interim guidance for clinical laboratories, containing real-time reverse-transcription polymerase chain reaction (rRT-PCR) primers and probes, which shall be used for purposes of epidemic surveillance (14). Contextually, the WHO has also released another interim guidance for laboratory diagnosis of infection, which includes recommendations for sample collection, handling and storage, as well as indications on 2019-nCoV detection, accompanied by a list of currently available protocols (15).

A reasonable question would hence follow after the onset of this novel epidemic challenge, the third $\mathrm{CoV}$ outbreak in less than 20 years, i.e., which lessons shall we learn from the 2019-nCoV outbreak? The first and most obvious issue, is that infectious diseases will be probably included among the most important health hazards that we will need to face in the foreseeable future. Although the WHO estimates that global mortality for infectious and parasitic diseases will decrease by more than half by the year 2060 (i.e., from $9.7 \%$ in 2016 to $3.9 \%$ in 2060) (16), epidemiology cannot reliably 


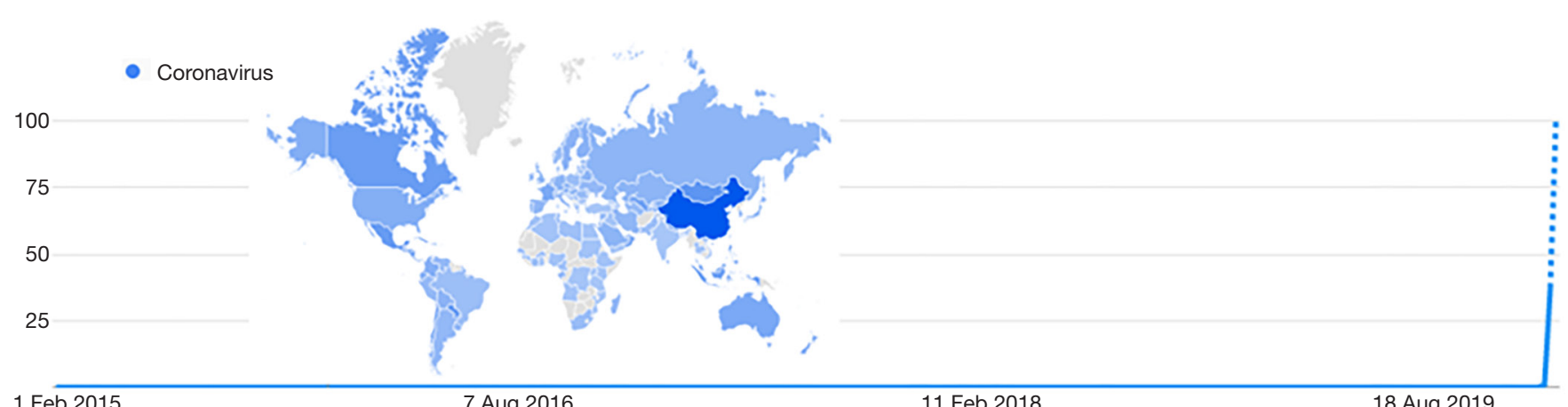

1 Feb 2015

7 Aug 2016

11 Feb 2018

18 Aug 2019

Figure 2 Output of an electronic search in Google Trends using the keyword "coronavirus".

predict the risk of emerging zoonotic diseases such as those caused by RNA recombination of $\mathrm{CoV}$. Antimicrobial resistance is another growing issue, which has been rated by the $\mathrm{WHO}$ as an increasingly serious threat to global public health, since it will challenge effective prevention and treatment of the ever-increasing array of infections caused by viruses, bacteria and other microorganisms (17). These two aspects shall hence contribute to raise public amplified that the worldwide resources spent for prevention, diagnosis and management of infectious diseases shall be magnified, rather than reduced. The efficient diagnosis of these severe viral infections is another relevant matter, since timely identification would allow rapid isolation and clinical management of diseased patients. Serology testing may be a good option for surveillance or investigational scopes, but not for diagnostic purposes, where molecular tests remain the gold standard. Molecular biology is a rapidly evolving enterprise. The development and implementation of new diagnostic tests, mostly encompassing rRT-PCR assays, is now very fast and straightforward, thus reaffirming the central role of laboratory diagnostics throughout the clinical reasoning (18). This would be especially important considering that widespread testing during the early phase of an outbreak could be really effective to attenuate virus spread. For example, robust diagnostic methodology has recently been developed and deployed for use in public health laboratory settings in Europe (19).

A final issue concerns the public information on this and other recent viral outbreaks. The results of a search in Google Trends using the keyword "CoV" is shown in Figure 2. Since the Google Trends score reflects the popularity of search queries in Google across various geographical regions and using different languages (20), it can be clearly seen that the volume of Google searches for "CoV" during the past 5 years has increased by many orders of magnitude since the beginning of the $2019-\mathrm{nCoV}$ outbreak, especially in China. Rather understandably, the large volume of false information disseminated through social media networks is contributing to increase the risk of either dangerous underestimation or excessive concern (i.e., the so-called "cyber-chondria") about this infectious disease (21), as can be seen now from many posts in Facebook and Twitter. Public authorities and healthcare administrators shall hence be engaged in a thoughtful policy of reliable public information, aimed at preventing that misinformation will flourish and addressing people towards the reliable sources, where evidence-based (scientific) information can be garnered. National governments and healthcare authorities are also called to establishing narrower partnership and reinforced collaboration for facing emerging health challenges, whereby the recent 2019-nCoV outbreaks clearly demonstrates that a singlenation healthcare issue may soon become a worldwide problem.

\section{Acknowledgments}

None.

\section{Footnote}

Conflicts of Interest: The authors have no conflicts of interest to declare.

Ethical Statement: The authors are accountable for all aspects of the work in ensuring that questions related to the accuracy or integrity of any part of the work are appropriately investigated and resolved. 


\section{References}

1. Zhu N, Zhang D, Wang W, et al. A Novel Coronavirus from Patients with Pneumonia in China, 2019. N Engl J Med 2020. [Epub ahead of print].

2. Perlman S. Another Decade, Another Coronavirus. N Engl J Med 2020. [Epub ahead of print].

3. GenBank. 2019-nCoV Sequences (Wuhan coronavirus). Available online: https://www.ncbi.nlm.nih.gov/nuccore/ MN908947. Last accessed, January 29, 2020.

4. Zhu N, Zhang D, Wang W, et al. A Novel Coronavirus from Patients with Pneumonia in China, 2019. N Engl J Med 2020. [Epub ahead of print].

5. Ji W, Wang W, Zhao X, et al. Homologous recombination within the spike glycoprotein of the newly identified coronavirus may boost cross-species transmission from snake to human. J Med Virol 2020. [Epub ahead of print].

6. Phan LT, Nguyen TV, Luong QC, et al. Importation and Human-to-Human Transmission of a Novel Coronavirus in Vietnam. N Engl J Med 2020. [Epub ahead of print].

7. Munster VJ, Koopmans M, van Doremalen N, et al. A Novel Coronavirus Emerging in China - Key Questions for Impact Assessment. N Engl J Med 2020. [Epub ahead of print].

8. World Health Organization. Novel Coronavirus (2019$\mathrm{nCoV}$ ) situation reports. Available online: https://www. who.int/emergencies/diseases/novel-coronavirus-2019/ situation-reports. Last accessed, January 28, 2020.

9. Lippi G, Plebani M. The novel coronavirus (2019-nCoV) outbreak: think the unthinkable and be prepared to face the challenge. Diagnosis (Berl) 2020. [Epub ahead of print].

10. Huang C, Wang Y, Li X, et al. Clinical features of patients infected with 2019 novel coronavirus in Wuhan, China. Lancet 2020. [Epub ahead of print].

11. Wang W, Tang J, Wei F. Updated understanding of the outbreak of 2019 novel coronavirus (2019-nCoV) in Wuhan, China. J Med Virol 2020. [Epub ahead of print].

Cite this article as: Mattiuzzi C, Lippi G. Which lessons shall we learn from the 2019 novel coronavirus outbreak? Ann Transl Med 2020;8(3):48. doi: 10.21037/atm.2020.02.06
12. Chen N, Zhou M, Dong X, et al. Epidemiological and clinical characteristics of 99 cases of 2019 novel coronavirus pneumonia in Wuhan, China: a descriptive study. Lancet 2020. Doi: 10.1016/S01406736(20)30211-7

13. Li Q, Guan X, Wu P et al. Early Transmission Dynamics in Wuhan, China, of Novel Coronavirus-Infected Pneumonia. N Engl J Med 2020. [Epub ahead of print].

14. Centers for Disease Control and Prevention (CDC). 2019 Novel Coronavirus Real-time RT-PCR Resources. Available online: https://www.cdc.gov/coronavirus/2019ncov/downloads/rt-pcr-panel-primer-probes.pdf. Last accessed, January 28, 2020.

15. World Health Organization. Laboratory testing for 2019 novel coronavirus (2019-nCoV) in suspected human cases. Interim guidance. Available online: https://www.who.int/ health-topics/coronavirus/laboratory-diagnostics-fornovel-coronavirus. Last accessed, January 28, 2020.

16. World Health Organization. Projections of mortality and causes of death, 2016 to 2060. Available online: https://www.who.int/healthinfo/global_burden_disease/ projections/en/. Last accessed, January 28, 2020.

17. World Health Organization. Antimicrobial resistance. Available online: https://www.who.int/antimicrobialresistance/en/. Last accessed, January 28, 2020.

18. Plebani M, Laposata M, Lippi G. A manifesto for the future of laboratory medicine professionals. Clin Chim Acta 2019;489:49-52.

19. Corman VM, Landt O, Kaiser M, et al. Detection of 2019 novel coronavirus (2019-nCoV) by real-time RTPCR. Euro Surveill 2020. doi: 10.2807/1560-7917. ES.2020.25.3.2000045.

20. Lippi G, Cervellin G. Is digital epidemiology reliable?insight from updated cancer statistics. Ann Transl Med 2019;7:15.

21. Ventola CL. Social media and health care professionals: benefits, risks, and best practices. P T 2014;39:491-520. 